\title{
OPEN A LC-MS method for 25-hydroxy-vitamin D3 measurements from dried blood spots for an epidemiological survey in India
}

Rashmi Lote-Oke ${ }^{1}$, Jwala Pawar ${ }^{1}$, Shriram Kulkarni ${ }^{1}$, Prasanna Sanas ${ }^{1}$, Neha Kajale ${ }^{1}$, Ketan Gondhalekar ${ }^{1}$, Vaman Khadilkar ${ }^{1}$, Siddhesh Kamat ${ }^{2}$ \& Anuradha Khadilkar ${ }^{1 凶}$

Vitamin $\mathrm{D}$, a secosteroid, plays an important role in several physiological processes, and its deficiency can lead to numerous pathophysiological conditions in humans. The primary objective of this study was to develop and validate the robustness of a mass spectrometry-based method capable of quantifying $25(\mathrm{OH}) \mathrm{D} 3$ for an upcoming epidemiological survey in India and to pilot test it on healthy volunteers. We first describe the development and validation of various experimental parameters that ascertain the robustness and reliability of 25-hydroxy-vitamin D3 (25(OH)D3) extractions and quantitative measurements from Dried Blood Spot (DBS) samples, where we used eight disks of $3 \mathrm{~mm}$ each, punched from the circular spot covering the entire circumference of the spot. Next, we conducted a pilot study, comparing $25(\mathrm{OH}) \mathrm{D} 3$ levels from serum and DBS samples from 45 participants using a protocol developed for specifically this purpose. We found that the mean $25(\mathrm{OH})$ D3 concentrations in DBS samples were comparable to the serum levels $(P>0.05)$. In summary, our extraction and LC-MS protocol for quantitative $25(\mathrm{OH}) \mathrm{D} 3$ measurements are robust and reproducible, and will serve as an invaluable tool for upcoming epidemiological surveys in India and perhaps around the world.

Vitamin $\mathrm{D}$ is a secosteroid that plays a significant role in bone mineralization and remodelling by regulating the calcium and phosphorus homeostatic balance ${ }^{1}$. Recent reports suggest that it also plays an important role in several other vital physiological functions such as the protection against respiratory tract infections, muscle contractions and nerve conduction ${ }^{2}$. Humans typically obtain vitamin D from two sources: exposure to sunlight which converts 7-dehydrocholesterol (7DHC) present in epidermal layer of skin into cholecalciferol (vitamin D3) and consumption of vitamin D rich foods such as certain fatty fish, shitake mushrooms and yeast which provide ergocalciferol (vitamin D2) ${ }^{3}$. In humans, both ergocalciferol and cholecalciferol are hydroxylated by hepatic hydroxylases to 25 hydroxy-vitamin $\mathrm{D}[25(\mathrm{OH}) \mathrm{D} 2$ and $25(\mathrm{OH}) \mathrm{D} 3)]^{4}$. Being the primary storage form with longer half-life, the concentration of 25-hydroxy-vitamin D is measured to determine the physiological vitamin D status ${ }^{5}$.

Vitamin D bioavailability from commonly consumed foods is very low in most populations, particularly when the foods are not fortified. Therefore, the primary source for acquiring vitamin D in most populations is through sunlight exposure, and hence understandably, populations with limited sunlight availability (especially in winter seasons) are more prone to its deficiency. Interestingly, vitamin D deficiency has also been reported in countries located in lower latitudes, where there is no scarcity of sunlight, with India being one of these nations. The majority of the Indian population lives in the region of ample sunshine throughout the year (8.4 to $37.6^{\circ}$ north latitude), and despite this, studies have reported vitamin D deficiency in different age groups and geographical locations of India ${ }^{4}$.

Despite the lack of consensus within the scientific community on desired physiological concentrations of vitamin $\mathrm{D}$, the most commonly accepted definition for vitamin $\mathrm{D}$ deficiency indicates a blood concentration less than $20 \mathrm{ng} / \mathrm{mL}$ ( $50 \mathrm{nmol} / \mathrm{l}$ ) in adults ${ }^{6}$. While there are several studies that have consistently reported high

${ }^{1}$ Hirabai Cowasji Jehangir Medical Research Institute, Pune, India. ${ }^{2}$ Department of Biology, Indian Institute of Science Education and Research, Pune, India. ${ }^{\circledR e m a i l}$ : anuradhavkhadilkar@gmail.com 
prevalence of vitamin D deficiency globally, comparable population scale studies in an Indian population, are to the best of our knowledge, not performed, and hence unavailable.

Thus, the primary purpose of the current study was to develop an efficient and robust epidemiological protocol for assessment of vitamin D in various representative groups of the Indian population using dried blood spots (DBS). Dried blood spotting is minimally invasive and a resource friendly alternative to serum/plasma samples, especially in large epidemiological studies. Being minimally invasive it is also the method of choice for paediatric and geriatric populations ${ }^{7}$. The specific objectives our study were (1) to develop and validate the robustness of a mass spectrometry-based method capable of quantifying $25(\mathrm{OH}) \mathrm{D} 3$ that we intend using for subsequent epidemiological studies, (2) to assess $25(\mathrm{OH}) \mathrm{D} 3$ concentrations in serum and DBS (using the newly developed method) in a pilot sample of healthy adult volunteers.

\section{Materials and methods}

Chemicals. Analytical standards for 25(OH)D3 were purchased from Sigma-Aldrich. LC-MS grade acetonitrile, methanol, and hexane were purchased from J.T. Baker (Thermo Fisher Scientific). Double deionized water was purchased from HiMedia Laboratories. Stock solutions of $25(\mathrm{OH}) \mathrm{D} 3$, and $\left[{ }^{2} \mathrm{H}_{6}\right]-25(\mathrm{OH}) \mathrm{D} 3$ were prepared at a concentration of $1 \mathrm{mg} / \mathrm{mL}$ in ethanol and stored at $-80^{\circ} \mathrm{C}$ until use.

Blood sampling and spotting. Healthy adult volunteers (age: 22 to 48 years) were requested to provide blood samples for the study, as per a protocol which was approved by the Institutional Ethics CommitteeJehangir Clinical Development Centre Private Limited. We confirm that all the experiments in this study are in accordance with Declaration of Helsinki, Institutional Ethics committee and Institutional scientific committee. All volunteers provided an informed consent to participate in the study. Volunteers were examined by a physician on site to confirm absence of any disease before sampling was performed. Samples were collected according to the guidelines in the Declaration of Helsinki for preparation of vitamin D free artificial blood and for assessment of vitamin D from serum and DBS.

Preparation of vitamin D free artificial blood. Blood samples ( $3 \mathrm{~mL}$ each) were collected in plain and EDTA tubes from 25 healthy volunteers in a community health camp, pooled and centrifuged at $2500 \mathrm{rpm}$ for $10 \mathrm{~min}$ at room temperature to separate the samples into serum/plasma and cellular components. The serum was aliquoted into separate tubes and conserved for preparation of vitamin $\mathrm{D}$ free serum. Cellular components (red blood cells, leukocytes and thrombocytes) were washed with $25 \mathrm{~mL}$ phosphate-buffered saline (PBS) solution ( $\mathrm{pH}$ 7.4), followed by centrifugation at $2500 \mathrm{rpm}$. This step was repeated 5 times. The pooled serum was then mixed thoroughly with activated charcoal $(0.14 \mathrm{gm} / 10 \mathrm{~mL}$ of serum) by shaking at $280 \mathrm{rpm}$ for $8 \mathrm{~h}$. The mixture was centrifuged at $10,000 \mathrm{rpm}$ for $25 \mathrm{~min}$. The supernatant was separated, the centrifugation step was repeated, and the remaining serum was collected by filtration. The filtered charcoal treated serum $(2 \mathrm{~mL})$ was stored at $-20{ }^{\circ} \mathrm{C}$ until use ${ }^{8}$. The PBS treated cellular components and the charcoal treated serum were mixed together in a ratio of 37:63 (v/v) based on the natural composition of female whole blood (using mean adult female haematocrit values from a previous study) resulting in $10 \mathrm{~mL}$ of artificial vitamin $\mathrm{D}$-free whole blood as a homogenous mixture of serum and blood cells ${ }^{5}$.

Blood spot (DBS) sample preparation. From each of the 45 study participants, intravenous samples were collected (approximately 3-4 mL) using plain and Heparin tubes. Immediately after collection, $3 \mathrm{~mL}$ whole blood from both tubes was aliquoted in an appropriate tube and Calcifediol- $\mathrm{d}_{6}(15 \mu \mathrm{L})$ was added to it to achieve a concentration of $5 \mathrm{ng} / \mathrm{mL}$, ensuring thorough mixing. DBS cards were prepared by pipetting $55 \mu \mathrm{L}$ of whole blood with $\left[{ }^{2} \mathrm{H}_{6}\right]-25(\mathrm{OH}) \mathrm{D} 3$ on each spot. The blood spots were then allowed to dry at room temperature for $2 \mathrm{~h}$ and were subsequently transferred to light protective storage polythene bags along with a desiccant. The bags were stored at $-20^{\circ} \mathrm{C}$ until extraction. $500 \mu \mathrm{L}$ of whole blood was separately aliquoted and used for Haematocrit analysis using Yumizen H 500 (Horiba, India). Approximately $30 \mathrm{~min}$ after the addition of $\left[{ }^{2} \mathrm{H}_{6}\right]-25(\mathrm{OH}) \mathrm{D} 3$, the plain and heparin tubes were centrifuged for $10 \mathrm{~min}$ at $2500 \mathrm{rpm}$ and aliquots of $500 \mu \mathrm{L}$ of serum/plasma were prepared. All the aliquots were flash frozen in liquid nitrogen and stored at $-80^{\circ} \mathrm{C}$ freezer until extraction. Samples were neither subjected to prolonged light exposure nor multiple freeze/thaw cycles.

Matrix matched calibration. For serum. The charcoal treated serum was used for matrix calibration. Each tube containing $0.5 \mathrm{~mL}$ charcoal treated serum was spiked with $25(\mathrm{OH}) \mathrm{D} 3$ spanning a concentration range of $2.25,11.25,22.5,67.5,112.5,225 \mathrm{ng} / \mathrm{mL}$.

For DBS. The blank DBS cards (Whatman filter paper number 903) were prepared by spotting $55 \mu \mathrm{L}$ of artificial blood in each spot. Eight discs of $3 \mathrm{~mm}$ diameter were punched into a $2 \mathrm{~mL}$ Eppendorf tube from the DBS. Each tube was spiked with $10 \mu \mathrm{L}$ of $25(\mathrm{OH}) \mathrm{D} 3$ spanning a concentration range of $6.4,9.6,25.6,51.2,102.4,153.6$ and $204.8 \mathrm{ng} / \mathrm{mL}$.

Extraction of vitamin D. For serum. The serum extraction method was a modification of a previously described method ${ }^{9}$. To the $500 \mu \mathrm{L}$ serum sample a mixture of $200 \mu \mathrm{L}$ of Acetonitrile: Methanol (70:30) was added. The tube was briefly vortexed and then sonicated for $30 \mathrm{~min}$ (Labman Scientic Instruments, Chennai, India). After sonication, $1 \mathrm{~mL}$ hexane was added followed by brief vortexing and centrifugation at $10,000 \mathrm{rpm}$ for $10 \mathrm{~min} .900 \mu \mathrm{L}$ of hexane was carefully transferred to a fresh tube without disturbing the phase separation. 
The hexane was evaporated to dryness using nitrogen concentrator (KeMi scientific, Pune India). The residue was reconstituted in $200 \mu \mathrm{L}$ of methanol followed by vortexing to ensure uniform mixing.

For DBS. DBS extraction method was a modification of a previously described method ${ }^{10}$. A circular disc of $3 \mathrm{~mm}$ diameter (corresponding to $3.2 \mu \mathrm{L}$ of whole blood) was punched from a spot on the DBS card using a semi-automated puncher (Horizon Speciality, USA). For the assay, 8 discs from each participant were used (corresponding to $25.6 \mu \mathrm{L}$ of whole blood). The punch was cleaned with $70 \%$ ethanol prior to and after taking sample from each study participant. The discs were placed into $2 \mathrm{~mL}$ Eppendorf tubes along with $250 \mu \mathrm{L}$ of water. Each tube was vortexed for $30 \mathrm{~s}$ and then sonicated for $1 \mathrm{~h}$ using a sonicator (Labman Scientific Instruments, Chennai, India). $250 \mu \mathrm{L}$ of methanol was added to each tube and the tubes were sonicated for another $30 \mathrm{~min}$. Subsequently, $500 \mu \mathrm{L}$ of hexane was added to each tube followed by centrifugation at $10,000 \mathrm{rpm}$ for $10 \mathrm{~min}$. Approximately $400 \mu \mathrm{L}$ of hexane was carefully aspirated in a fresh tube without disturbing the phase separation and evaporated to dryness using nitrogen concentrator (Kemi Scientific, Pune India). The residue was reconstituted in $25 \mu \mathrm{L}$ of methanol followed by vortexing to ensure uniform mixing.

LC-MS/MS. The LC-MS system used was Shimadzu 8045 triple quadrupole mass spectrometer (MS) with the electrospray ionization (ESI) source in positive ion polarity mode fitted with Nexera X2 LC-30A UHPLC system. Data were collected in the multiple reaction monitoring (MRM) mode monitored for various vitamin D components in the extract using the following MS parameters: oven temperature $30{ }^{\circ} \mathrm{C}$, interface temperature $298{ }^{\circ} \mathrm{C}$, desolvation line temperature $196{ }^{\circ} \mathrm{C}$, nebulizing gas flow $3.0 \mathrm{~L} / \mathrm{min}$, heating gas flow $10.0 \mathrm{~L} / \mathrm{min}$, drying gas flow 18.0 L/min, IG vacuum 1.7e - $003 \mathrm{~Pa}$, PG vacuum 6.9e+001 Pa, CID Gas 230 KPa. Each vitamin D3 species and internal standard was identified and quantified using three MRM transitions reported previously, that for the various species were: $(\mathrm{i}) 25(\mathrm{OH}) \mathrm{D} 3=401.2 \rightarrow 383.3,401.2 \rightarrow 365.4,401.2 \rightarrow 159.1$; (ii) vitamin D3 $=385.2 \rightarrow 259.2$, $385.2 \rightarrow 241.0,385.2 \rightarrow 107.3$; and (iii) the internal standard $\left[{ }^{2} \mathrm{H}_{6}\right]-25(\mathrm{OH}) \mathrm{D} 3=407.5 \rightarrow 389.5,407.5 \rightarrow 371.0$, $407.5 \rightarrow 107.0^{9}$. Isolation and separation of analytes by chromatography was achieved by using a Shimadzu Premier C18 $150 \mathrm{~mm} \times 2.1 \times 3 \mu \mathrm{m}$ column without derivatization. A typical LC-MS consisted of $10 \mathrm{~min}$, with mobile phase $\mathrm{A}=$ water $+0.1 \%$ formic acid, and $\mathrm{B}=$ methanol $+0.1 \%$ formic acid, with a flow rate of $400 \mu \mathrm{L} / \mathrm{min}$, and had the following gradient sequence: (i) equilibration with solvent B at $90 \%$ from 0.0 to $1.4 \mathrm{~min}$, (ii) linear increase in solvent B from 90 to $100 \%$ from 1.5 to $4 \mathrm{~min}$, and (iii) equilibration and washing with solvent B at $90 \%$ from 4.1 to $10 \mathrm{~min}$. MS acquisition was stopped after $5 \mathrm{~min}$ of the LC gradient, and the remaining eluent was directed to waste collection system.

Statistical analysis. SPSS software was used for data analysis (version 26 0.0, IBM statistics data editor, IBM Corp., released 2017. Armonk, NY). Variables to be tested were assessed for normality using KolmogorovSmirnov KS test. Depending on the normality, appropriate tests e.g. independent sample t-test for normal and Wilcoxon Signed Rank Test (for non-normal variables) were applied. For categorical variables, Chi square test was used for reporting associations between two categorical variables. Bland Altman plot was constructed to assess differences between the $25(\mathrm{OH}) \mathrm{D} 3$ concentrations assessed using the two methods, i.e. from serum and DBS. Level of significance was set at $\mathrm{P}<0.05$.

Ethics declaration. Ethical Approval was obtained from the Institutional ethics committee (Jehangir Clinical Development Centre Private Limited, Approval dated 21/06/2016). We confirm that all the experiments in this study are in accordance with Declaration of Helsinki, Institutional Ethics committee and scientific committee. All volunteers provided an informed consent to participate in the study.

\section{Results}

Validation of LC-MS/MS method. The LC-MS/MS method described here was validated based on the procedures described in the 'Bioanalytical method validation Guidance for Industry' of the U S Food and Drug Administration ${ }^{11}$. A schematic workflow of the steps involved in the method validation is presented in Fig. 1. Since this assay was adapted in part from a previously reported and validated method ${ }^{10}$, only a partial validation of this method using the fit for purpose concept was performed here.

The method was selectivity tested by comparing chromatograms from five independent samples of vitamin D free serum and artificial DBS with and without spiking $\left[{ }^{2} \mathrm{H}_{6}\right]-25(\mathrm{OH}) \mathrm{D} 3$ or $25(\mathrm{OH}) \mathrm{D} 3$. As the samples contained endogenous $25(\mathrm{OH}) \mathrm{D} 3$, area ratios of qualifier MRM transition $401.2 \rightarrow 383.3$ to quantifier MRM transition $401.2 \rightarrow 365.4$ were calculated and used for the assessment of selectivity for the detection of $25(\mathrm{OH})$ D3. Representative spectra for the qualifier and quantifier MRM for both the analyte $25(\mathrm{OH}) \mathrm{D} 3$, and the internal standard $\left[{ }^{2} \mathrm{H}_{6}\right]-25(\mathrm{OH}) \mathrm{D} 3$ are presented in Fig. 2. Carry over was assessed by measuring blank samples after analysis of every high-content sample, and no significant carryover $(<1 \%)$ was observed in our method. Linearity and dynamic range of this LC-MS/MS method were also determined by analysis of calibration samples (Fig. 3), and were found to pass set experimental thresholds (Table 1). The limit of quantification (LOQ) and the limit of detection (LOD) for 25(OH)D3 were set to a signal to noise ratio threshold of 10 and 5 respectively, and were found to be approximately 10 and $5 \mathrm{ng} / \mathrm{mL}$ respectively (Fig. 3). Differences between nominal concentrations and calculated concentrations were determined and expressed in percentage. Deviations of the measured values from nominal values should be $\leq 20 \%$ for the LOQ (LOQ) and $\leq 15 \%$ for the remaining calibration points. At least $75 \%$ of the calibration levels on each day need to meet these criteria. As calibration was performed with endogenous samples, the following concentration range was tested: $2 \cdot 25-225 \mathrm{ng} / \mathrm{mL}$ calculated as serum concentrations. Details of the parameters assessed, along with their acceptance criteria, and the experimental outcome for this method for quantification of 25(OH)D3 can be found in Table 1. 


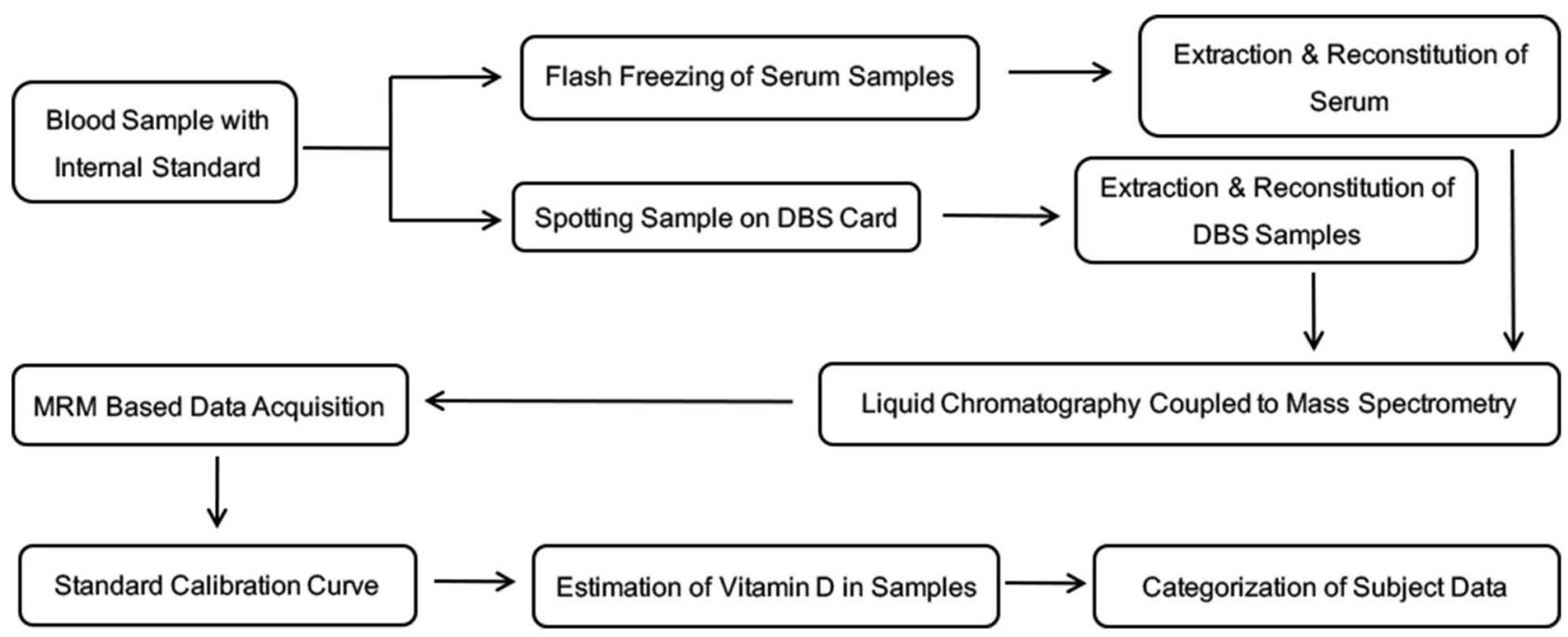

Figure 1. Sample analysis workflow. Blood collection in study participants, the serum was separated from blood and flash freezing was carried out in liquid nitrogen, uncoagulated blood was spread on DBS card and allowed to dry completely at room temperature. Both samples were extracted on the same day using respective extraction protocols followed by LCMS analysis in Shimadzu 8045. Matrix matched calibration was performed using charcoal treated serum for serum samples and vitamin D free artificial blood for DBS samples and standard calibration curves were generated for both. The circulating vitamin D levels were estimated based on these standard calibration curves.

Assessing the matrix effect and inter-day variance of the method. Although accepted as the gold standard, $\mathrm{LC}-\mathrm{MS} / \mathrm{MS}$ analysis of $25(\mathrm{OH}) \mathrm{D} 3$ from serum is not a universal assay and variability in sample preparation, chromatographic separation and ionization/fragmentation have been reported ${ }^{12}$. Matrix effects (ion suppression/ion enhancement) are recognized as one of the major potential sources of errors in serum LC-MS/MS analysis of $25(\mathrm{OH}) \mathrm{D} 3$, and hence it has been recommended that the matrix effects for any LC-MS/MS measurement of 25(OH)D3 should be evaluated thoroughly ${ }^{12}$. Also, the ionization efficiency of $25(\mathrm{OH}) \mathrm{D} 3$ is known to be low and in the absence of derivatization relatively larger volume of serum/plasma $(0.1-0.5 \mathrm{~mL})$ need to be used. Similarly, for DBS samples it has been reported that the composition of blood and its volume dramatically affects metabolite measurements from DSB samples. Hence, to overcome any matrix effects, matrix matched calibration using artificially prepared $25(\mathrm{OH}) \mathrm{D} 3$ free blood is recommended ${ }^{5}$.

For the current study, we performed matrix matched calibration using charcoal treated serum for serum samples and artificially prepared blood using same charcoal treated serum for DBS samples. Considering the fact that $25(\mathrm{OH}) \mathrm{D} 3$ is hydrophobic in nature and is tightly bound to vitamin $\mathrm{D}$ binding protein(s), its extraction efficiency from serum may be affected. To ensure the maximum extraction efficiency in serum samples, we used a twopronged strategy. Firstly, after separation of serum, the samples were flash frozen using liquid nitrogen. The goal of flash freezing was to minimize any potential biochemical reactions in serum that may result in metabolism/ degradation of serum $25(\mathrm{OH}) \mathrm{D} 3$. Secondly, in a subset of the experimental samples $(\mathrm{n}=31)$, a known concentration of internal standard $\left(\left[{ }^{2} \mathrm{H}_{6}\right]-25(\mathrm{OH}) \mathrm{D} 3\right)$ was spiked in to establish an extraction recovery factor. Addition of internal standard was followed by separation as serum. The peak areas of the internal standard $\left(\left[{ }^{2} \mathrm{H}_{6}\right]-25(\mathrm{OH})\right.$ D3) were obtained at the end of extraction and were used to calculate the \% recovery of $\left[{ }^{2} \mathrm{H}_{6}\right]-25(\mathrm{OH}) \mathrm{D} 3$ in each sample. To ensure a uniform recovery calculation, same procedure was used to calculate recovery from corresponding DBS samples. The mean recovery for serum sample was found to be $79.03 \%$ while that for the corresponding DBS sample was found to be $69.72 \%$. The $\%$ mean recovery was used to calculate the recovery factor (100/mean \% recovery), and these were set at 1.27 and 1.43 for serum and DBS samples respectively.

For DBS samples, the measured 25(OH)D3 concentration needed to be normalized to its \% haematocrit. The $\%$ haematocrit for the artificial blood used for calibration was 37\%, and the following formula (Eq. 1) was used for normalizing the 25(OH)D3 concentrations for DBS samples that were used in these calibration experiments. For experimental (subject) DBS samples, the 25(OH)D3 concentration was further corrected for individual haematocrit (HCT) value for each participant by applying the respective correction factor (Eq. 2). Hence, for the experimental (subject) DBS samples, the final 25(OH)D3 concentration was eventually obtained by multiplying Eq. (1) by the individual HCT correction factor that was obtained from Eq. (2).

$$
\begin{gathered}
{[25(\mathrm{OH}) \mathrm{D} 3]_{\text {normalised }}=[25(\mathrm{OH}) \mathrm{D} 3]_{\text {measured }} \times(100-37) / 100} \\
\text { Individual HCT Correction Factor }=(100-37) /(100-\% \text { HCT for participant })
\end{gathered}
$$

Next, for assessing accuracy and inter-day variance of this method, three samples representing low, medium and high concentrations with five aliquots of each were analysed on 3 different days for both serum and DBS samples. The artificial blood with $37 \%$ haematocrit using charcoal treated serum was used as a blank for both 

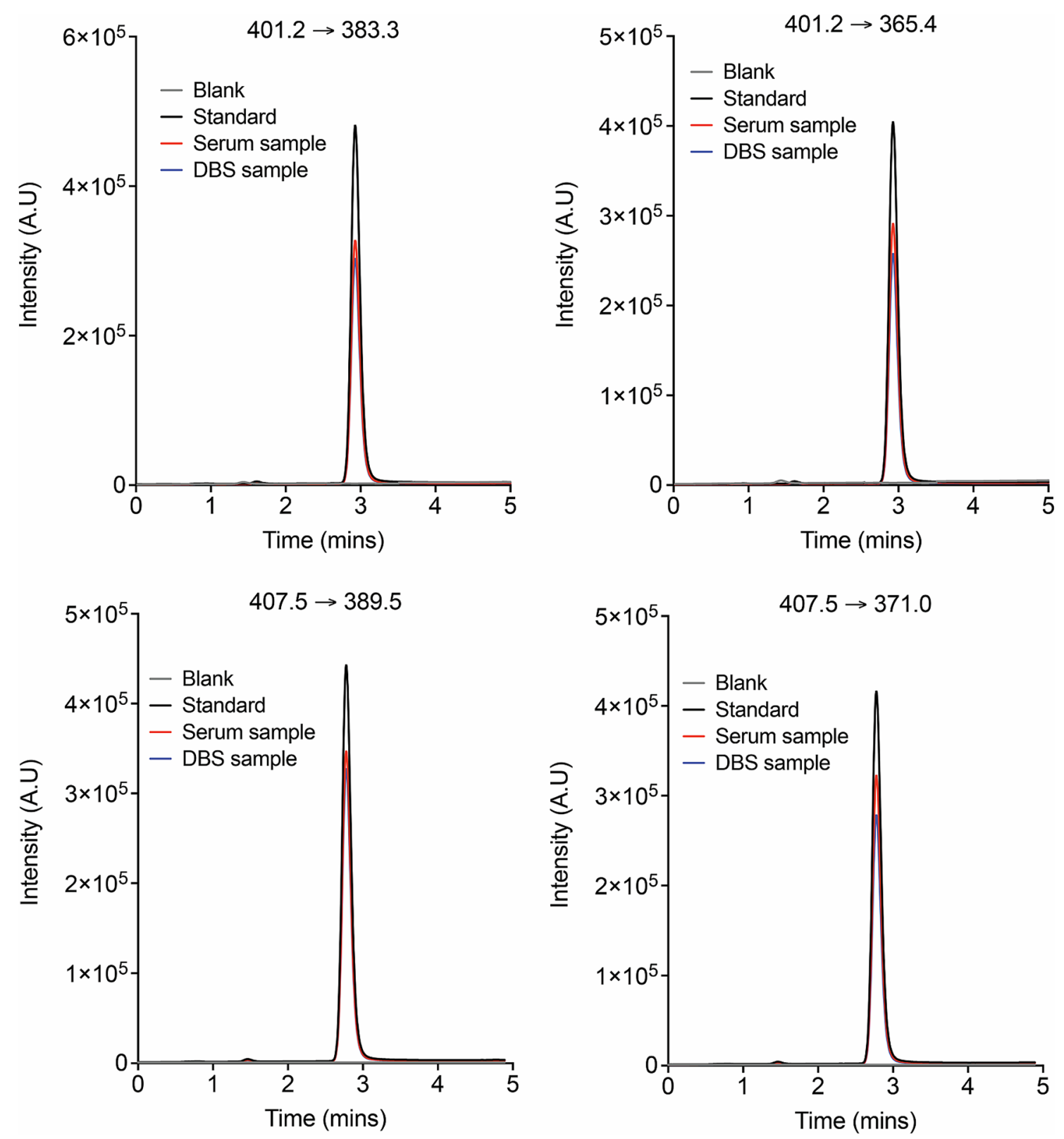

Figure 2. Representative spectra for the qualifier and quantifier MRM of 25(OH)D3 (analyte; $\mathrm{m} / \mathrm{z}=401.2$ ), and $\left[{ }^{2} \mathrm{H}_{6}\right]-25(\mathrm{OH}) \mathrm{D} 3$ (internal standard; $\mathrm{m} / \mathrm{z}=407.5$ ).
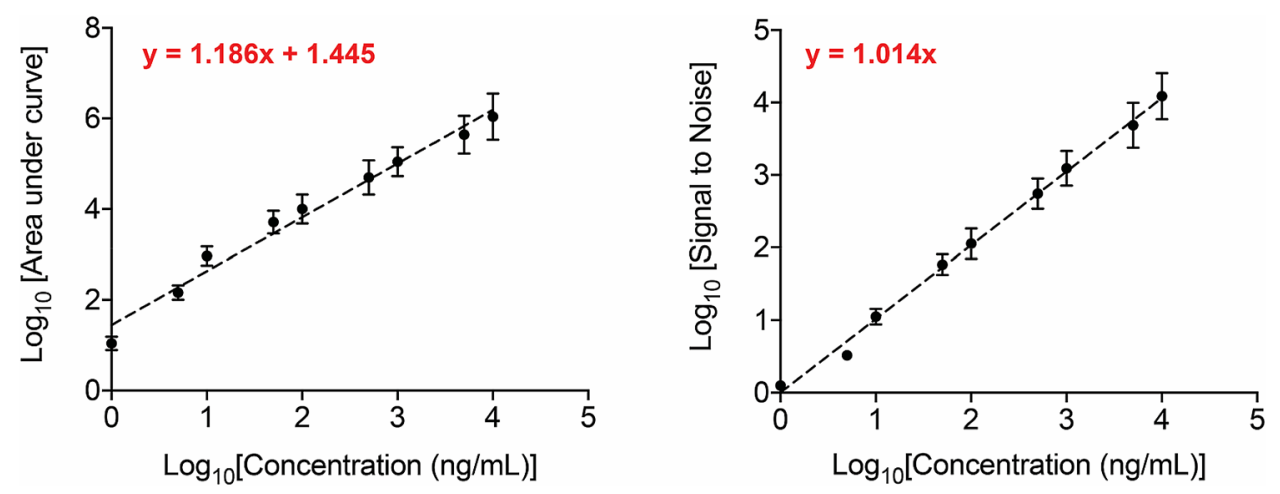

Figure 3. Plots displaying linearity, dynamic range, and signal to noise ratio for 25(OH)D3 measurements using the LC-MS method. 


\begin{tabular}{|c|c|c|c|}
\hline Validation element & Description & Acceptance criteria & Results \\
\hline Selectivity & $\begin{array}{l}\text { Minimum interference in the detection of } \\
\text { analyte of interest ensured by assessing matrix } \\
\text { effect on ion suppression, ion enhancement or } \\
\text { extraction efficiency }\end{array}$ & $\begin{array}{l}\text { Blank and zero calibrators should be free of } \\
\text { interference at the retention times of the analyte } \\
\text { and IS } \\
\text { Spiked samples should be } \pm 20 \% \text { LOQ } \\
\text { The IS response in blank should not exceed } 5 \% \\
\text { of the average IS response of the calibrators and } \\
\text { QCs }\end{array}$ & Pass \\
\hline Carry-over & Response of analyte due to the previous injection & Carry over should not exceed $20 \%$ of LOQ & Pass \\
\hline Accuracy and precision & $\begin{array}{l}\text { To ensure the extraction protocol is efficient and } \\
\text { reproducible }\end{array}$ & $\begin{array}{l} \pm 20 \% \text { of nominal concentration at LOQ } \\
\text { and } \pm 15 \% \text { for the others } \\
\% \text { RSD } \pm 20 \% \text { at LOQ and other levels }\end{array}$ & $\begin{array}{l}< \pm 20 \% \text { RSD at LOQ and }< \pm 15 \% \text { RSD at other } \\
\text { concentrations }\end{array}$ \\
\hline Linearity \& dynamic range & $\begin{array}{l}\text { Concentration range accepted in the particular } \\
\text { study }\end{array}$ & $\begin{array}{l}\text { Non zero calibration should be } \pm 15 \% \text { of nominal } \\
\text { concentrations and for LOQ calibrators should } \\
\text { be } \pm 20 \% \\
75 \% \text { and a minimum of six non zero calibrator } \\
\text { levels should meet the above criteria in each } \\
\text { calibration run } \\
3 \text {-orders of linear range }\end{array}$ & Pass \\
\hline
\end{tabular}

Table 1. Description of the parameters for validation of the LC-MS/MS method for quantification of 25(OH) D3. $L O Q$ limit of quantification, $R S D$ residual standard deviation.
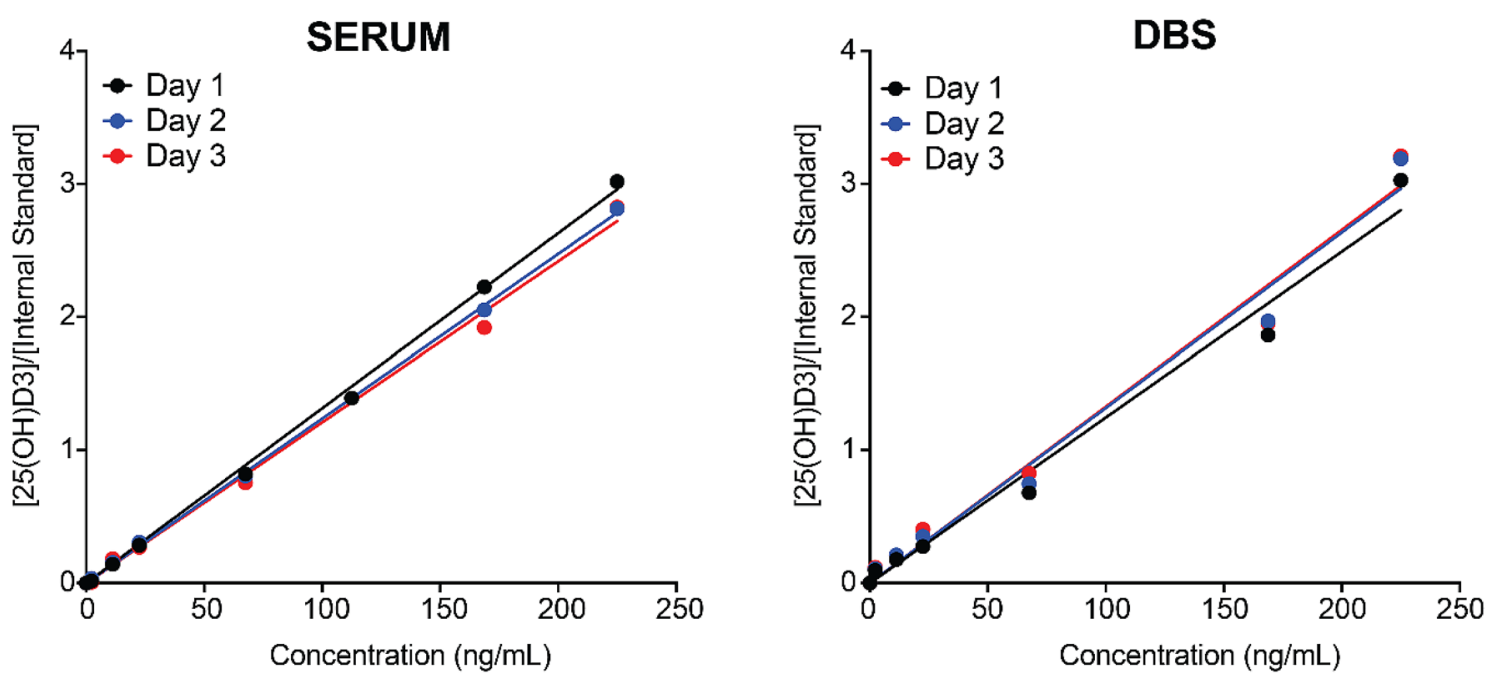

Figure 4. Inter-day accuracy and precision for serum and DBS samples.

serum and DBS samples. To estimate inter-day precision, triplicates of each sample were analysed on three different days, and these were found to be identical in this method (Fig. 4).

Human subject data analysis. Finally, to validate the robustness of our LC-MS/MS method in measuring 25(OH)D3 from human samples, we decided to perform a small pilot study in healthy human volunteers. In this pilot study, serum and DBS samples were extracted and analysed from 45 healthy volunteers, with a mean age $\sim 37$ years, mean body mass index $=24.5 \mathrm{~kg} / \mathrm{m}^{2}($ males $=23)$. Mean vitamin $25(\mathrm{OH}) \mathrm{D} 3$ concentrations in serum and DBS samples from these human subjects were $47 \pm 19$ and $44 \pm 19 \mathrm{ng} / \mathrm{mL}$ respectively. As the primary aim of our study was to develop an efficient epidemiological protocol for assessment of 25(OH)D3 concentrations in a representative Indian population, we classified the samples as vitamin D deficient (vitamin $25(\mathrm{OH})$ D3 levels $<20 \mathrm{ng} / \mathrm{mL}$ ), insufficient (vitamin $25(\mathrm{OH}) \mathrm{D} 3<30 \mathrm{ng} / \mathrm{mL}$ ) and vitamin D sufficient (vitamin $25(\mathrm{OH})$ D3 $>30 \mathrm{ng} / \mathrm{mL}$ ) by both the serum and DBS methods (Fig. 5). We found that there were no significant differences in the proportion of subjects classified as deficient, insufficient and sufficient by using either serum or DBS samples for the assessment of $25(\mathrm{OH}) \mathrm{D} 3$ (Fig. 5) $(\mathrm{P}>0.1)$. Further, we observed that by using this method to assess 25(OH)D3 concentrations in serum or DBS samples, the Pearson Chi square values were similar to each other $\left(\Psi^{2}=8.63, \mathrm{df}=4, \mathrm{P}=0.07\right)$, thus validating the robustness of our method. In accordance with a previously reported analysis for such data, we also assessed these findings statistically using a Bland-Altman plot, and found the serum and DBS data to be in good accordance, and within experimental error limits 9 .

\section{Discussion}

In humans approximately $95 \%$ of vitamin D3 is synthesized in epidermal keratinocytes from $7 \mathrm{DHC}^{13}$. The B-ring of 7DHC absorbs UVB radiation in the range of 280-320 nm which breaks the bond between C9 and C10 resulting in formation of pre-vitamin D3. Thermal isomerization converts pre-vitamin D3 to D $3^{14}$. The vitamin 


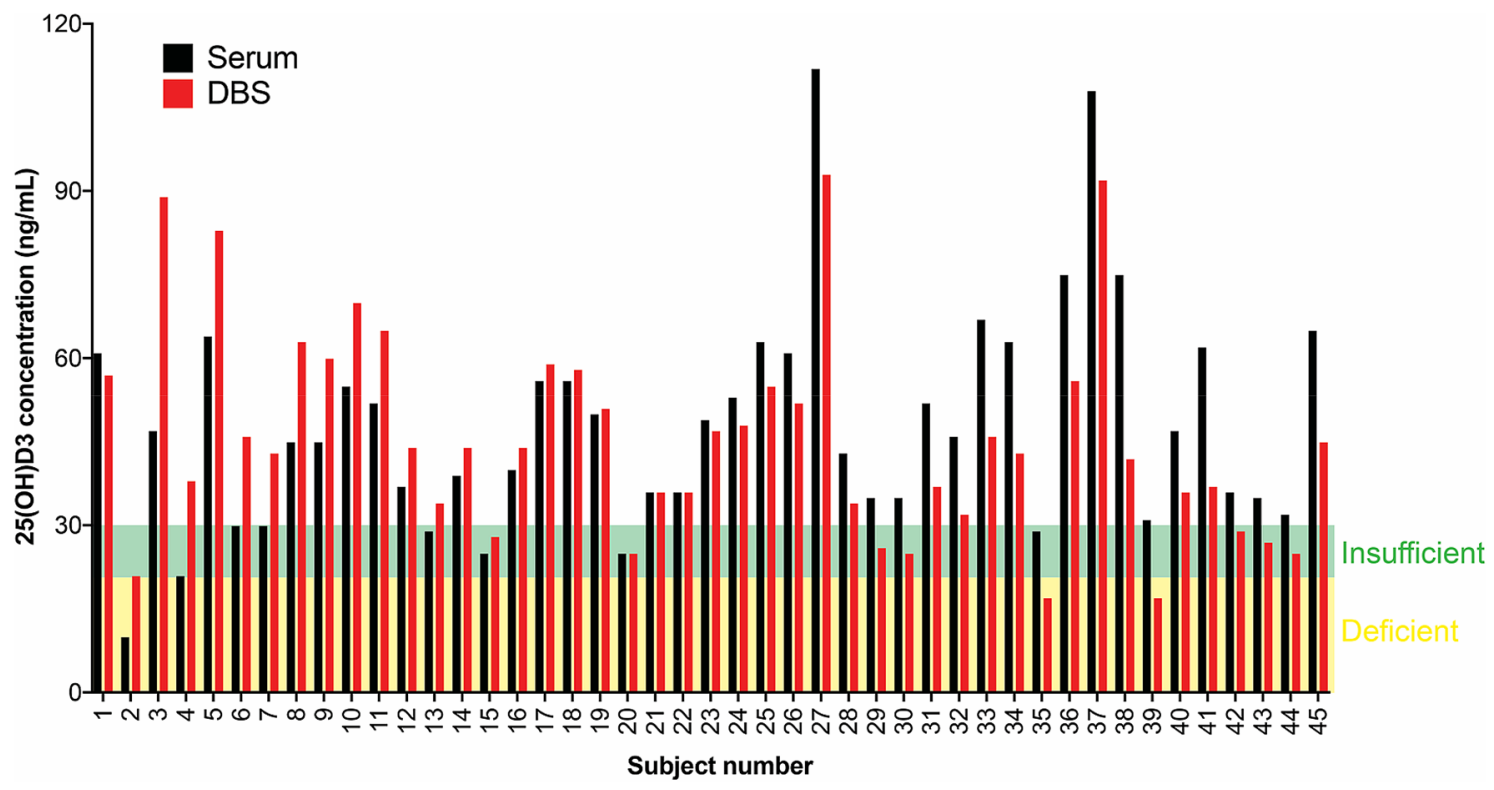

Figure 5. Pilot Study to analyse circulating vitamin D levels using DBS samples.

D3 is hydroxylated at 25th position by hepatic CYP27A1 or CYP2R1 to produce $25(\mathrm{OH}) \mathrm{D} 3)$. The 25(OH)D3 is subsequently hydroxylated at $\mathrm{C} 1 \alpha$ in kidneys and other peripheral tissues by CYP27B1 to form $1,25(\mathrm{OH})_{2} \mathrm{D} 3$ which is the biologically active form of vitamin $\mathrm{D}^{14}$. Another cytochrome P450 enzyme (CYP11A1) catalyses sequential hydroxylation of D3 side chain to form several vitamin D metabolites such as $20(\mathrm{OH}) \mathrm{D} 3,22(\mathrm{OH})$ D3, $[2 \mathrm{O}, 22(\mathrm{OH}) 2 \mathrm{D} 3],[20,23(\mathrm{OH}) 2 \mathrm{D} 3]$ and $[17,20,23(\mathrm{OH}) \mathrm{D} 3]^{15}$. Among these, $20(\mathrm{OH}) \mathrm{D} 3$ is the predominant metabolite of this pathway, that can be detected in serum at an approximately 20 times lower concentration compared to $\mathrm{D} 3^{13}$.

Prolonged exposure to UVB can cause photo-isomerization of pre-vitamin D3 to tachysterol3 (T3) which undergo UVB driven conversion to sterol3 (L3). Formation of T3 and L3 prevents excessive formation of previtamin D3 and thus protects from D3 intoxication. Recently it has been reported that L3 undergoes CYP11A1 mediated hydroxylation and the hydroxylated lumisterol derivatives provide protection against UVB damage. The hydroxylumisterol derivatives are reported to be present in serum suggesting additional biological activity ${ }^{16,17}$. The CYP11A1 derivatives can be further hydroxylated at C1 $\alpha$ by CYP27A1, CYP24A1, CYP11A1, CYP2R1 and/ or CYP2A4. It has also been reported that CYP11 A1 derived $20,23(\mathrm{OH})_{2} \mathrm{D} 3$ and $1,25(\mathrm{OH})_{2} \mathrm{D} 3$ induce some overlapping gene expression patterns in keratinocytes ${ }^{18}$. CYP11A1 derived compounds i.e. both the D3 and L3 derivatives display anti-proliferative, anti-inflammatory, anti-cancer and pro-differentiation properties ${ }^{17}$.

Physiologically and clinically, vitamin D status refers to the concentration of hydroxylated D3 and D2 together, and the most widely accepted biochemical marker for assessing systemic vitamin D status is the total serum 25 -hydroxyvitamin $\mathrm{D}[25(\mathrm{OH}) \mathrm{D}]$ concentration $^{19}$. For the past decade, quantitative vitamin $\mathrm{D}$ analysis from serum/plasma using LC-MS has been considered the gold standard given its sensitivity, precision and lowered susceptibility to matrix effect compared to cell-based assays ${ }^{10}$. Previously published literature has reported that measurement of $25(\mathrm{OH}) \mathrm{D} 3$ from DBS using LC-MS/MS is a valid alternative to conventional methods of quantifying vitamin D metabolites from plasma/serum samples. However, being a relatively new and less explored method of analysis, vitamin D analysis from DBS samples shows considerable variability and optimization studies regarding this method are lacking in literature ${ }^{20}$. The primary goal of our study was to optimize and standardize a method for analysis of 25(OH)D3 using DBS for an upcoming epidemiological study in representative Indian population. For any clinical (or epidemiological) assay, the most important performance parameters to be considered are sensitivity, specificity, throughput and labour for sample preparation ${ }^{21}$. Derivatization i.e. modification of original chemical structure of analyte with diverse tags ${ }^{22}$ is commonly used to improve the sensitivity of the vitamin $\mathrm{D}$; however, it makes the assay laborious and may hamper accuracy by forming multiple stereoisomers ${ }^{10}$. Hence, here, we focused on developing an efficient, high throughput method for screening vitamin D deficiency without using chemical derivatization.

It has been estimated that in the Indian population, sunlight exposure leading to indigenous synthesis of D3 is the primary determinant of vitamin D status as it contributes to $>90 \%$ of the total $25(\mathrm{OH}) \mathrm{D}$ concentration ${ }^{23}$. It has also been reported that, in a population that consumes food items not being fortified with vitamin $\mathrm{D}, 25(\mathrm{OH})$ D2 levels were below the detectable limit and hence its assessment of agreement between DBS and serum samples cannot be implemented in an Indian population ${ }^{24}$. Therefore here, we decided to assess the relative concentration of only $25(\mathrm{OH}) \mathrm{D} 3$ in DBS and serum, and validate a method for the same. There is a common agreement in the scientific community that $25(\mathrm{OH}) \mathrm{D} 3$ is a difficult analyte to quantitate and thus methods for the same are sparse in literature ${ }^{25}$. As a result, the implementation of novel LC-MS/MS based methods in a clinical lab is challenging due to the lack of agreement in serum and DBS samples. However, for an epidemiological study, with the primary aim of reporting prevalence of vitamin D deficiency, the goal should be to accurately report the 
vitamin D deficiency/sufficiency status compared to a diagnostic protocol where the aim is to derive the method for analysis of vitamin D in DBS that can closely reflect the physiological vitamin D status as closely possible to serum/plasma samples. The LC-MS/MS we report here, does all the above, and fits the bill for us to implement this for our upcoming epidemiological survey.

Despite its robustness, our method still has a few limitations. Here, we have used a C18 stationary phase and this cannot separate isomeric and isobaric vitamin D metabolites. For example, a C18 column can achieve only partial separation of 25(OH)D3 and $25(\mathrm{OH}) \mathrm{D} 2$ as well as between $25(\mathrm{OH}) \mathrm{D} 3$ and its C3-epimer ${ }^{7}$. As a result, our method may sometimes lead to the overestimation of $25(\mathrm{OH}) \mathrm{D}$ concentrations. The accuracy of estimation of vitamin D requires selection of columns that offer high chromatographic selectivity prior to MS identification. However, this strategy sacrifices high throughput due to additional time requirement. Pentafluorophenyl (PFP) column which requires comparatively shorter run time can perhaps be used ${ }^{7}$ but will be substantially more expensive, additionally, it may not be economically feasible for a population scale study. Lastly, a 2-step derivatization i.e. a Diels-Alder reaction with 4-phenyl-1,2,4-triazoline-3,5-dione (PTAD) followed by acetylation, may be used ${ }^{7}$. However, derivatization can compromise accuracy because of its own limitations as discussed in the previous section. Selection of $\mathrm{C} 18$ column was a decision in interest of high throughput and cost-effective method. We acknowledge that because of these limitations, the protocol may be used for a population based epidemiological study rather than for diagnostic purposes.

\section{Data availability}

The datasets generated during and/or analysed during the current study are available from the corresponding author on reasonable request.

Received: 29 July 2020; Accepted: 28 October 2020

Published online: 16 November 2020

\section{References}

1. Holick, M. F. The role of vitamin D for bone health and fracture prevention. Curr. Osteoporos. Rep. 4, 96-102 (2006).

2. Endocrine, P. \& Hospital, J. Vitamin D Deficiency in Indian Adolescents. 1-2 (2010).

3. Rola, R., Kowalski, K., Bieńkowski, T., Kołodyńska-Goworek, A. \& Studzińska, S. Development of a method for multiple vitamin D metabolite measurements by liquid chromatography coupled with tandem mass spectrometry in dried blood spots. Analyst 144, 299-309 (2019).

4. Adela, R. et al. Lower vitamin D metabolites levels were associated with increased coronary artery diseases in type 2 diabetes patients in India. Sci. Rep. 6 (2016).

5. Müller, M. J., Stokes, C. S. \& Volmer, D. A. Quantification of the $3 \alpha$ and $3 \beta$ epimers of 25 -hydroxyvitamin D3 in dried blood spots by LC-MS/MS using artificial whole blood calibration and chemical derivatization. Talanta 165, 398-404 (2017).

6. Holick, M. F. The vitamin D deficiency pandemic: Approaches for diagnosis, treatment and prevention. Rev. Endocr. Metab. Disord. 18, 153-165 (2017).

7. Van Den Ouweland, J. M. W., Vogeser, M. \& Bächer, S. Vitamin D and metabolites measurement by tandem mass spectrometry. Rev. Endocr. Metab. Disord. 14, 159-184 (2013).

8. Carter, P. Preparation of ligand-free human serum for radioimmunoassay by adsorption on activated charcoal. Clin. Chem. 24, 362-364 (1978).

9. Gören, A. C., Bilsel, G., Bilsel, M., Tak, T. U. B. İ. \& Gebze-kocaeli, P. B. Rapid and simultaneous determination of 25-OH-vitamin D 2 and D 3 in human serum by LC/MS/MS : Validation and uncertainty assessment. 1, 1-9 (2007).

10. Makowski, A. J., Rathmacher, J. A., Horst, R. L. \& Sempos, C. T. Simplified 25-hydroxyvitamin D standardization and optimization in dried blood spots by LC-MS/MS. J. AOAC Int. 100, 1328-1336 (2017).

11. FDA, F. and D. A. Bioanalytical method validation guidance. Food Drug Admin. 1043, 25 (2018)

12. Couchman, L., Benton, C. M. \& Moniz, C. F. Variability in the analysis of 25 -hydroxyvitamin D by liquid chromatography-tandem mass spectrometry: The devil is in the detail. Clin. Chim. Acta 413, 1239-1243 (2012).

13. Slominski, A. T. et al. In vivo evidence for a novel pathway of vitamin D 3 metabolism initiated by P450scc and modified by CYP27B1. FASEB J. 26, 3901-3915 (2012).

14. Slominski, A. T. et al. Detection of novel CYP11A1-derived secosteroids in the human epidermis and serum and pig adrenal gland. Sci. Rep. 5, 1-12 (2015).

15. Slominski, A. T. et al. RORo: And ROR 7 are expressed in human skin and serve as receptors for endogenously produced noncalcemic 20-hydroxy- and 20,23-dihydroxyvitamin D. FASEB J. 28, 2775-2789 (2014).

16. Slominski, A. T. et al. Characterization of a new pathway that activates lumisterol in vivo to biologically active hydroxylumisterols. Sci. Rep. 7, 1-17 (2017).

17. Slominski, A. T. et al. Photoprotective properties of vitamin D and lumisterol hydroxyderivatives. Cell Biochem. Biophys. 78, $165-180(2020)$.

18. Slominski, A. T. et al. Differential and overlapping effects of 20,23(OH)2 D3 and 1,25(OH)2 D3 on gene expression in human epidermal keratinocytes: Identification of AHR as an alternative receptor for 20,23(OH)2 D3. Int. J. Mol. Sci. 19, 1-35 (2018).

19. Couchman, L. \& Moniz, C. F. Analytical considerations for the biochemical assessment of vitamin D status. Ther. Adv. Musculoskelet. Dis. 9, 97-104 (2017).

20. Hoeller, U. et al. Application of dried blood spots to determine vitamin D status in a large nutritional study with unsupervised sampling: The Food4Me project. Br. J. Nutr. 115, 202-211 (2016).

21. Higashi, T., Shimada, K. \& Toyooka, T. Advances in determination of vitamin D related compounds in biological samples using liquid chromatography-mass spectrometry: A review. J. Chromatogr. B Anal. Technol. Biomed. Life Sci. 878, 1654-1661 (2010).

22. Zhu, Y., Deng, P. \& Zhong, D. Derivatization methods for LC-MS analysis of endogenous compounds. Bioanalysis 7, 2557-2581 (2015).

23. Indian Council of Medical Research. Final draft nutrient requirements and recommended dietary allowances for Indians. A Report of the Expert Group of the Indian Council of Medical Research. Expert Gr. Indian Council Med. Res. 1-334 (2009).

24. Larkin, E. K. et al. Agreement of blood spot card measurements of vitamin D levels with serum, whole blood specimen types and a dietary recall instrument. PLoS One 6 (2011).

25. Carter, G. D. 25-Hydroxyvitamin D: A difficult analyte. Clin. Chem. 58, 486-488 (2012). 


\section{Acknowledgements}

This study was funded by way of an educational grant from Zuventus Healthcare Ltd, and a DST-FIST Infrastructure Development grant (SR/FST/LSII-043/2016) to the Biology Department of IISER Pune.

\section{Author contributions}

Concept Development, Consenting and Health check was performed by A.V.K., V.V.K., N.A.K. Data collection, method development and data analysis were carried out by R.O., S.K., J.P., S.K., P.S., K.G. and A.V.K. All authors contributed to the manuscript writing.

\section{Competing interests}

The authors declare no competing interests.

\section{Additional information}

Correspondence and requests for materials should be addressed to A.K.

Reprints and permissions information is available at www.nature.com/reprints.

Publisher's note Springer Nature remains neutral with regard to jurisdictional claims in published maps and institutional affiliations.

(1) Open Access This article is licensed under a Creative Commons Attribution 4.0 International License, which permits use, sharing, adaptation, distribution and reproduction in any medium or format, as long as you give appropriate credit to the original author(s) and the source, provide a link to the Creative Commons licence, and indicate if changes were made. The images or other third party material in this article are included in the article's Creative Commons licence, unless indicated otherwise in a credit line to the material. If material is not included in the article's Creative Commons licence and your intended use is not permitted by statutory regulation or exceeds the permitted use, you will need to obtain permission directly from the copyright holder. To view a copy of this licence, visit http://creativecommons.org/licenses/by/4.0/.

(c) The Author(s) 2020 